University of Nebraska - Lincoln

DigitalCommons@University of Nebraska - Lincoln

1983

\title{
A Simplified Method to Calculate Failure Times in Fault-Tolerant Systems
}

Sharad C. Seth

University of Nebraska-Lincoln, seth@cse.unl.edu

Lester Lipsky

University of Nebraska-Lincoln

Follow this and additional works at: https://digitalcommons.unl.edu/csearticles

Part of the Computer Sciences Commons

Seth, Sharad C. and Lipsky, Lester, "A Simplified Method to Calculate Failure Times in Fault-Tolerant Systems" (1983). CSE Journal Articles. 38.

https://digitalcommons.unl.edu/csearticles/38

This Article is brought to you for free and open access by the Computer Science and Engineering, Department of at DigitalCommons@University of Nebraska - Lincoln. It has been accepted for inclusion in CSE Journal Articles by an authorized administrator of DigitalCommons@University of Nebraska - Lincoln. 


\section{A Simplified Method to Calculate Failure Times in Fault-Tolerant Systems}

\section{SHARAD C. SETH AND LESTER LIPSKY}

\begin{abstract}
A simplified method is presented to calculate moments of failure time and residual lifetime of a fault-tolerant system. The method is based on recent results in queueing theory. Its effectiveness is illustrated by considering a dual repairable system from the literature.
\end{abstract}

Index Terms-Failure-time distribution, fault-tolerant systems, mean time to failure (MTTF), mean residual life (MRL), renewal theory.

\section{INTRODUCTION}

Fault-tolerant computing systems are commonly modeled as continuous-time Markov chains for reliability analysis [1], [2]. Systems both with and without repair may be modeled this way for calculation of reliability, mean time to failure (MTTF), mission time, mean time to repair, mean residual lifetime (MRL), and other important measures. The model may also be extended to calculate performance-related measures for gracefully degrading systems [3]. The purpose of this correspondence is to call to attention recent results [4], [5] which simplify calculation of MTTF and higher moments of the time to system failure. The particular case of MTTF was also obtained by Laprie and is reported in [2]; however, it is argued that the higher moments are of equal interest, e.g., in calculating MRL and its variance. With the reported result, it becomes quite feasible to run parametric studies on these other measures as well. This is illustrated by considering an example from the literature [6]. It is interesting to note that the calculation of MTTF for the example reveals an unnoticed error (albeit, a minor one) in this classic paper, suggesting the difficulty of computation by the traditional method. First this method will be briefly reviewed to provide a basis for comparison.

\section{Traditional Method of Calculating MTTF and HIGHER MOMENTS}

Assume that the Markov model has $m+1$ states labeled $S_{0}, S_{1}$, $S_{2}, \cdots, S_{m}$ where $S_{0}$ represents the system failure state. Let $p_{i}(t)$ be the probability that the system is in state $S_{i}$ at time $t$, given the initial system state at $t=0$. Further, assume that for $i \neq j, \lambda_{i j} \Delta t$ is the probability of making a transition from state $S_{i}$ to $S_{j}$ in a small time interval $\Delta t$. Then, for continuous Markov chains, the $p_{i}(t)$ 's are solutions of the following vector differential equation?:

$$
\boldsymbol{p}^{\prime}(t)=-\boldsymbol{p}(t) Q
$$

Where $Q$ is an $(m+1) \times(m+1)$ matrix such that

$$
Q_{i j}=-\lambda_{i j}, \quad i \neq j
$$

and

$$
Q_{i i}=\left(\lambda_{i 0}+\lambda_{i 1}+\cdots+\lambda_{i m}\right)
$$

Let $t_{f}$ denote the time to system failure. This represents the time to first transition to the state $S_{0}$ which can, therefore, be made an absorbing state without affecting the distribution of $t_{f}$. With this assumption, the matrix $Q$ has the form

$$
\left[\begin{array}{ll}
0 & 0 \\
q^{t} & q_{G}
\end{array}\right]
$$

where $Q_{G}$ is the $(m \times m)$ submatrix of $Q$ corresponding to the operational ("good") states of the system. Since the rows of $Q$ must sum to zero, we have

$$
\boldsymbol{q}^{T}=-Q_{G} \mathbf{e}^{T}
$$

where $\boldsymbol{e}$ is the $m$-dimensional vector with all 1's. Now, by definition, the moments of $t_{f}$ are given by

$$
t_{f}^{n}=\int_{0}^{\infty} t^{n} p_{0}^{\prime}(t) d t, \quad n \geq 1
$$

(Note that MTTF $=t_{f}^{1}$.) Also, from (1) and (2),

$$
p_{0}^{\prime}(t)=-p_{G}(t) q^{T}
$$

and

$$
\boldsymbol{p}_{G}^{\prime}(t)=-\boldsymbol{p}_{G}(t) Q_{G}
$$

where $\boldsymbol{p}=\left(p_{0}, p_{G}\right)$. Under the reasonable assumption that $V=Q_{G}^{-1}$ exists [that is, the system eventually reaches the failure state irrespective of the initial probability vector $\left.p_{G}(0)\right],(6)$ has the solution

$$
p_{G}(t)=p_{G}(0) \exp \left(-Q_{G} t\right) V
$$

where $p_{G}(0) e^{T}=1$. Equation (7) can be substituted in (5) to obtain $p_{0}^{\prime}(t)$. Then the integral in (4) can be evaluated to yield the $n$th moment of $t_{f}$. However, even for a small number of states, the procedure becomes computationally expensive because of the need to compute the matrix function exp $\left(-Q_{G} t\right)$. Further, for hand computation, necessary in parametric studies such as [6], this lengthy and tedious process is prone to errors.

\section{A Simplified Method}

The basis for a simplified calculation of moments of $t_{f}$ can be found in equivalent results obtained recently by Carroll et al. [4] and by Neuts [5]. However, as these results appear outside the context of fault-tolerant computing, we will paraphrase them here in terms well understood within the field.

Recalling that $V$ denotes $Q_{G}^{-1}$, let us define

$$
\psi\left[V^{n}\right]:=p_{G}(0) V^{n} \mathrm{e}^{T} .
$$

Then it can be shown that

1 Vectors, denoted by boldface letters, are assumed to be row vectors; column vectors will be denoted explicitly as transposes of row vectors.
Manuscript received July 31, 1981; revised June 15, 1982.

The authors are with the Department of Computer Science, University of Nebraska, Lincoln, NE 68588. 


$$
\bar{t}_{f}^{n}=n ! \psi\left[V^{n}\right] .
$$

The special case of this formula for $\operatorname{MTTF}(n=1)$ was also derived by Laprie solving the forward Kolmogorov equations; it appears in [2].

The MTTF concerns the mean lifetime of a system from its first startup. What may be of equal importance is the time to failure for a system given that it has already been operational for an indefinite (unknown or averaged over) period. This is commonly known as the mean residual lifetime (MRL) and has been shown to be (see [7, p. 173])

$$
\mathrm{MRL}=\frac{\bar{t}_{f}^{2}}{2 \bar{t}_{f}}
$$

From (9) and (10),

$$
\mathrm{MRL}=\frac{\psi\left[V^{2}\right]}{\psi[V]}
$$

which is computationally quite efficient.

It is worth emphasizing that only for exponential decay rates is the MRL equal to the MTTF. This can be seen by rewriting (10) assuming that $\sigma^{2}=\bar{t}_{f}^{2}-\bar{t}_{f}^{2}$ and $c^{2}=\sigma^{2} / \bar{t}_{f}^{2}$ :

$$
\begin{aligned}
\mathrm{MRL} & =\frac{\bar{t}_{f}^{2}}{2 \bar{t}_{f}}=\frac{1}{2} \bar{t}_{f}+\frac{\sigma^{2}}{2 \bar{t}_{f}} \\
& =\frac{1}{2} \bar{t}_{f}\left(1+c^{2}\right) .
\end{aligned}
$$

Note that for exponential decay rate, $c=1$ and MRL $=$ MTTF. There are realistic situations for both $c^{2}>1$ and $c^{2}<1$. For instance, systems in which intrinsically faulty devices may occur (perhaps due to inadequate acceptance testing by the manufacturer) will have $c^{2}$ much greater than one, giving the behavior that if a device has already lasted one MTTF, it most surely was not one of the faulty types, and therefore will last much longer yet. On the other hand, multistage repair processes are examples where $c^{2}$ is much less than one, for here, if a long enough time has elapsed, the system must be in one of the later repair stages and will finish soon.

The matrix $V$ contains considerable information beyond that which is implied by (9). For instance, since (9) is valid for any initial vector $\boldsymbol{p}_{G}(0)$, by letting $\boldsymbol{p}_{G}(0)=\left(0,0, \cdots, 1_{i}, 0, \cdots, 0\right)$, it is seen that $\left(V \mathbf{e}^{T}\right)_{i}$ $=V_{i 1}+V_{i 2}+\cdots+V_{i m}$ is the mean time it will take the system to fail given that it is in state " $i$ " initially. Furthermore, it is known from renewal theory [4], [5] that the mean time the system will spend in state " $i$ " overall before failure is $\left(p_{G} V\right)_{i}$. That is, the system will go from one up state to another, returning to state " $i$ " an average of $\left(\boldsymbol{p}_{G} V\right)_{i} / t_{i}$ times before failure where $t_{i}=1 /\left(\lambda_{i 1}+\lambda_{i 2}+\cdots+\lambda_{i m}\right)$. It follows, then, that the conditional probability of finding the system in state " $i$," given that it has not yet failed and has been operational for an indefinite period, is

$$
\left(\boldsymbol{p}_{\tau}\right)_{i}=\frac{\left(\boldsymbol{p}_{G}(0) V\right)_{i}}{\psi[V]}
$$

Let $t_{\tau}$ be the random variable denoting the time to system failure given that it has already been operational for an indefinite period. Then, clearly, MRL is the first moment of $t_{\tau}$. Computation of the moments of $t_{\tau}$ is almost as easy as those of $t_{f}$. One need only observe that $\boldsymbol{p}_{\tau}$ plays the same role for residual time that $\boldsymbol{p}_{G}(0)$ plays for $t_{f}$. Thus,

$$
\mathrm{MRL}=\boldsymbol{p}_{\tau} V \boldsymbol{e}^{T}=\frac{\boldsymbol{p}_{G}(0) V^{2} e^{T}}{\psi[V]}=\frac{\psi\left[V^{2}\right]}{\psi[V]}
$$

which is identical to (11). In general, the $n$th moment for residual time is [compare to (9)]

$$
t_{\tau}^{n}=n ! \frac{\psi\left[V^{n+1}\right]}{\psi[V]}
$$

The formulas for variance and coefficient of variance for both failure and residual times are given below:

$$
\sigma_{f}^{2}=2 \psi\left[V^{2}\right]-(\psi[V])^{2}
$$

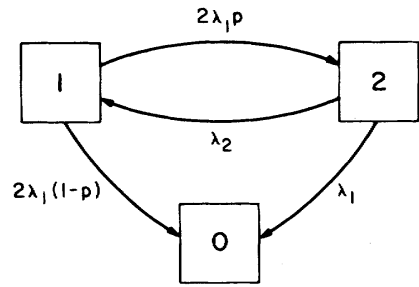

Fig. 1. Markov model for failure-time calculations for a duplicated repairable system [6]. Definition of states: 1-both system modules up; 2 -one module up, the other down, system is operational; 0 -system is down.

$$
\begin{aligned}
& \sigma_{\tau}^{2}=2 \frac{\psi\left[V^{3}\right]}{\psi[V]}-\left(\frac{\psi\left[V^{2}\right]}{\psi[V]}\right)^{2} \\
& c_{f}^{2}=2 \frac{\psi\left[V^{2}\right]}{(\psi[V])^{2}}-1 \\
& c_{\tau}^{2}=2 \frac{\psi\left[V^{3}\right] \psi[V]}{\left(\psi\left[V^{2}\right]\right)^{2}}-1 .
\end{aligned}
$$

\section{EXAMPLE}

Arnold [6] analyzes the effect of fault coverage on the reliability measures of a repairable system. Fig. 1 shows his Markov model for MTTF calculation for a duplicated repairable system. The parameters used in the figure are

$\lambda_{1}=$ failure rate of each module (time to failure and repair $\lambda_{2}=$ repair rate for a failed module are exponentially distributed) $p=$ fault coverages, that is, proportion of faults

from which the system can automatically recover.

The $Q$-matrix for the system can be directly written from Fig. 1:

$$
Q=\begin{aligned}
& 0 \\
& 1 \\
& 2
\end{aligned}\left[\begin{array}{ccc}
0 & 1 & 2 \\
0 & 0 & 0 \\
-2 \lambda_{1}(1-p) & 2 \lambda_{1} & -2 \lambda_{1} p \\
-\lambda_{1} & -2 \lambda_{2} & \left(\lambda_{1}+\lambda_{2}\right)
\end{array}\right]
$$

Clearly,

$$
\begin{aligned}
& V=\left[\begin{array}{cc}
2 \lambda_{1} & -2 \lambda_{1} p \\
-\lambda_{2} & \lambda_{1}+\lambda_{2}
\end{array}\right]^{-1} \\
& =\frac{1}{2 \lambda_{1}\left[\lambda_{1}+\lambda_{2}(1-p)\right]}\left[\begin{array}{cc}
\lambda_{1}+\lambda_{2} & 2 \lambda_{1} p \\
\lambda_{2} & 2 \lambda_{1}
\end{array}\right] .
\end{aligned}
$$

Let $q=1-p$ and $\delta=\lambda_{1} / \lambda_{2}$; then

$$
\lambda_{1} V=\frac{1}{2(q+\delta)}\left[\begin{array}{cc}
1+\delta & 2 \delta(1-q) \\
1 & 2 \delta
\end{array}\right] .
$$

Assuming that the system starts in state 1, we have, from (9) (in time units of $\left.1 / \lambda_{1}\right)$,

$$
\lambda_{1} \times \operatorname{MTTF}=\frac{[10]}{2(q+\delta)}\left[\begin{array}{cc}
1+\delta & 2 \delta(1-q) \\
1 & 2 \delta
\end{array}\right]\left[\begin{array}{l}
1 \\
1
\end{array}\right]=\frac{1+3 \delta-2 \delta q}{2(q+\delta)}
$$

Similarly, the second moment is

${ }^{2}$ Arnold appears to have made a computational error in calculating the MTTF-his numerator has the equivalent of $1+\delta(2+3 q)$. This does not, however, invalidate his results because he is mainly interested in the cases for which $\delta \ll 1$; under this condition, both expressions reduce to the same approximation. 


$$
\begin{aligned}
\lambda_{1}^{2} t_{f}^{2} & =\frac{2[10]}{4(q+\delta)^{2}}\left[\begin{array}{cc}
1+\delta & 2 \delta(1-q) \\
1 & 2 \delta
\end{array}\right]^{2}\left[\begin{array}{l}
1 \\
1
\end{array}\right] \\
& =\frac{1+6 \delta+7 \delta^{2}-2 \delta q(2+3 \delta)}{2(q+\delta)^{2}} .
\end{aligned}
$$

From (17) and (18), we have the mean residual life (in units of $\left.\lambda_{1}\right)$ :

$$
\begin{aligned}
\lambda_{1} \times \mathrm{MRL} & =\frac{1+6 \delta+7 \delta^{2}-2 \delta q(2+3 \delta)}{2(\delta+q)(1+3 \delta-2 \delta q)} \\
& \cong \frac{1+6 \delta}{2(\delta+q)(1+3 \delta)} \\
& \cong \frac{1+3 \delta}{2(\delta+q)}
\end{aligned}
$$

This shows that the effect of fault coverage on MRL is very similar to that on MTTF when both $\delta$ and $q$ are very small. Specifically, the effect becomes pronounced as the fraction of uncovered faults $q$ approaches the ratio $\delta$ of failure rate to repair rate. For this simple example system, the coefficients of variance $\left(c_{f}\right.$ and $\left.c_{\tau}\right)$, as calculated from (15), can be seen to be very close to unity. More interesting situations occur when failure or repair times are assumed not to be exponential. A detailed analysis of such systems will appear in a forthcoming paper under preparation by the authors.

\section{CONCLUSION}

The procedure described may be generalized to determine the mean time between arrivals to any state $s_{j}$ in the system by treating $s_{j}$ in the same way as the failure state; the vector $p_{G}(0)$ would have to be replaced by the vector of transition probabilities from $s_{j}$ to other states of the system. It is also possible to calculate the mean time between arrivals to a group of states basically by collapsing the group of states into a single "superstate." A special case of this situation occurs when MTTF is desired for a system modeled with multiple failure states. Neuts and Meier [8] consider a generalization of an entirely different kind. They discuss a duplicated system in which each "module" itself is described by a Markov process with an absorbing state.

\section{ACKNOWLEDGMENT}

The authors gratefully acknowledge the help given by A. van de Liefvoort during the preparation of this correspondence.

\section{REFERENCES}

[1] Y. W. Ng and A. Avizienis, "A unified reliability model for fault- tolerant computers," IEEE Trans. Comput., vol. C-29, pp. 1002-1011, Nov. 1980.

[2] A. Costes, J. E. Doucet, C. Landrault, and J. C. Laprie, "SURF: A program for dependability evaluation of complex fault-tolerant computing systems," in Proc. IIth Annu. Int. Symp. Fault-Tolerant Comput., June 1981, pp. 72-78.

[3] M. D. Beaudry, "Performance related reliability measures for computing systems," IEEE Trans. Comput., vol. C-27, pp. 540-547, June 1978.

[4] J. Carroll, A. van de Liefvoort, and L. Lipsky, "Solutions of $M / G / 1 / /$ $N$-type loops with extensions to $M / G / 1$ and $G I / M / 1$ queues," Oper. Res., vol. 30, pp. 390-414, May-June 1982.

[5] M. F. Neuts, "Renewal processes of phase type," Naval Res. Log. Quant., vol. 25 , pp. $445-454,1978$.

[6] T. F. Arnold, "The concept of coverage and its effect on the reliability model of a repairable system," IEEE Trans. Comput., vol. C-22, pp. 251-254, Mar. 1973.

[7] L. Kleinrock, Queueing Systems, Vol. 1. New York: Wiley, 1975.

[8] M. F. Neuts and K.S. Meier, "On the use of phase type distributions in reliability modelling of systems with two components," OR Spektrum, vol. 2, pp. 227-234, 1981. 